\title{
In Vivo Imaging With Two-Photon Microscope For Assessing Tumor Selective Binding Of Anti-CD137 Switch Antibody
}

\section{Chisato Kaneko}

Chugai Pharmaceutical Co., Ltd

Haruka Tsutsui

Chugai Pharmaceutical Co., Ltd

Kazuhisa Ozeki ( $\nabla$ ozekikzh@chugai-pharm.co.jp )

Chugai Pharmaceutical Co., Ltd

Masaki Honda

Chugai Pharmaceutical Co., Ltd

Kenta Haraya

Chugai Pharmaceutical Co., Ltd

Yoshinori Narita

Chugai Pharmabody Research Pte. Ltd.

Mika Kamata-Sakurai

Chugai Pharmaceutical Co., Ltd

Junichi Kikuta

Department of Immunology and Cell Biology

Mitsuyasu Tabo

Chugai Pharmaceutical Co., Ltd

Masaru Ishii

Osaka University

\section{Research Article}

Keywords: target, imaging, cytometry, binding

Posted Date: December 20th, 2021

DOI: https://doi.org/10.21203/rs.3.rs-1126148/v1

License: (c) (1) This work is licensed under a Creative Commons Attribution 4.0 International License.

Read Full License 


\section{Abstract}

STA551, a novel anti-CD137 switch antibody, binds to CD137 in an extracellular ATP (exATP) concentration dependent manner. Although STA551 was assumed to show higher target binding in tumor than normal tissues, quantitative detection of the target binding of switch antibody in vivo is technically challenging. In this study, we investigated the target binding of STA551 in vivo using intravital imaging with two-photon microscopy. Tumor-bearing human CD137 knock-in mice were intravenously administered $1 \mathrm{mg} / \mathrm{kg}$ of fluorescent-labeled antibodies at day 0 and 3 . Flow cytometry analysis of antibody-binding cells and intravital imaging using two-photon microscopy was conducted at day4. Higher CD137 expression in tumor than spleen was detected by flow cytometry analysis, and $\mathrm{T}$ cells and NK cells were major CD137 expressing cells. In the intravital imaging experiment, conventional and switch anti-CD137 antibody showed binding in tumor. However, in spleen, the fluorescence of switch antibody was much weaker than conventional anti-CD137 antibody and comparable with isotype control. In conclusion, we could assess switch antibody biodistribution in vivo through intravital imaging with two-photon microscopy. These results suggested that the tumor selective binding of STA551 leads to a wide therapeutic window and potent antitumor efficacy without systemic immune activation.

\section{Introduction}

CD137 is co-stimulatory receptor, and the stimulation of CD137 promotes T cell survival, proliferation and effecter function ${ }^{1,2}$. Several anti-CD137 agonist antibodies are being developed for the treatment of cancer $^{3}$. In clinical stages, two monoclonal antibodies, Urelumab (BMS-663513) and Utomilumab (PF05082566), have been administered to patients with tumors ${ }^{3}$. However, Urelumab caused serious hepatotoxicity in the phase I and II study ${ }^{4}$. Utomilumab showed lower toxicity but had less anti-tumor efficacy $^{5}$. To overcome the issues facing conventional anti-CD137 antibodies, we generated an antiCD137 agonist switch antibody, STA551 $1^{6}$. STA551 only binds to CD137 in the presence of ATP, but the binding is not detectable in the absence of ATP. A previous study reported that anti-CD137 switch antibody showed anti-tumor efficacy in various tumor models without systemic immune activation. Conventional anti-CD137 antibody induced splenomegaly, lymphadenopathy, and activation of T cells in normal tissues. On the other hand, anti-CD137 switch antibody did not show these responses in normal tissues. The data suggested that STA551 is a novel antibody which shows CD137 agonistic activity in tumor selectively.

Antitumor efficacy data in various mouse models and ex vivo analysis data suggested the selective binding of STA551 to CD137 in tumor, whereas quantitatively detecting conventional and switch antibody biodistribution in tumor and normal tissues under physiological condition has been a challenge ${ }^{6}$. ATP exists inside and outside the cell, and intracellular ATP concentration is much higher than extracellular ATP (exATP) ${ }^{7}$. Also, exATP levels are different between tissues. ExATP in tumor interstitial fluid is reported to be approximately $100 \mu \mathrm{M}$, whereas plasma and normal tissues contain low exATP levels (10-100 $\mathrm{nM})^{7-9}$. When animals are autopsied and tissues are sampled in ex vivo experiments, ATP concentration 
changes based on the physiological condition. ExATP and/or intracellular ATP might be degraded spontaneously or enzymatically, or intracellular ATP might be released from the cells during the process of ex vivo analysis. Thus, it is difficult to detect STA551 binding to the target quantitatively under physiological conditions using ex vivo analysis. Clarifying the minimal binding of STA551 in normal tissues in vivo would provide clear evidence of the less systematic immune activation of STA551. Therefore, we aimed to find a working method for elucidating STA551 biodistribution in vivo.

Confocal fluorescence microscopy has been widely used for observing cell physiology ${ }^{10}$. This tool allows us to observe high-resolution fluorescent images of cells and tissues. Since confocal microscopy uses single photon absorption processes to produce images, it can only visualize tissues at depths of up to $100 \mu \mathrm{m}^{11}$. In the last two decades, two-photon microscopy has been developed, which is an advanced form of microscopy which uses two-photon absorption processes to visualize images ${ }^{12,13}$. Since twophoton microscopy uses long wavelength lasers which has low energy, it causes low phototoxicity and allows long term imaging in a living animal. In addition, long wavelength light can penetrate deeper into tissues, allowing us to observe not only the surface but also deep inside tissues. Another advantage of this imaging technology is high resolution. Compared to PET and/or SPECT technology, two-photon imaging technology gives high-resolution images. High-resolution images allow us to observe detailed cell physiology, such cell morphology, movement, and cell-cell interaction ${ }^{14,15}$. Since two-photon microscopy is much better at intravital imaging, this technology is currently utilized to detect cell physiology in several organs including bone, brain and tumor ${ }^{16-19}$.

Intravital imaging using two-photon microscopes may be most appropriate way to detect switch antibody binding to the target cell for three reasons. First, two-photon microscopy can visualize deep inside various tissues, including tumor, spleen and lymph node. The difference in distribution between tumor and normal tissues should be detected. Second, we can obtain images in a living animal with physiological ATP concentrations in each tissue. Compared to ex vivo analysis, intravital imaging allows us to evaluate the antibody binding without injuring the cells or significantly interfering with ATP concentration. It was expected that the binding of switch antibody under physiological condition could be detected by intravital imaging. Third, high-resolution images allow us to observe antibody binding to the target cell.

In this study, we aimed to clarify the target binding of STA551 in tumor and normal tissues in vivo. First, we investigated CD137 expression in tumor-bearing human CD137 knock-in mice (hCD137 KI mice) and identified cell populations which express CD137. Second, we administered conventional CD137 agonistic antibody to tumor-bearing hCD137 KI mice and detected antibody-binding cells by flowcytometry. Finally, we administered switch or conventional CD137 agonistic antibody to mice and confirmed the different distributions of these antibodies to tumor and spleen using two-photon microscopy.

\section{Results}

To clarify the target binding of STA551 in tumor and normal tissues in vivo, we took three steps (Fig. 1). STA551 binds to CD137 of human and cynomolgus monkey but does not bind to murine CD137 ${ }^{6}$. 
Therefore, we used hCD137 KI mice ${ }^{6}$ to investigate STA551 binding to the target.

To investigate CD137 expression in hCD137 KI mice, we created a LLC1/OVA/hGPC3 model and detected CD137 expression by analyzing flowcytometry (Fig. 2). We first analyzed CD137 expression on CD45+ cells in tumor and spleen. CD $45^{+}$cells in tumor showed higher CD137 expression than in spleen (Fig. 2A). CD137 expression was observed in $2-8 \%$ of $C D 45^{+}$cells in tumor and in less than $2 \%$ of $C D 45^{+}$cells in spleen. Among CD $45^{+}$cells, CD8 ${ }^{+} \mathrm{T}$ cells and NK cells had high CD137 expression (Fig. 2B). CD137 expression on $\mathrm{CD} 4^{+} \mathrm{T}$ cells and $\mathrm{CD} 11 \mathrm{~b}^{+}$cells were also detected (Fig. 2B). These data suggested that anti-CD137 antibody would distribute more to tumor than to spleen and binds to CD $45^{+}$cells, especially CD8 ${ }^{+}$cells and NK cells.

In previous research, to evaluate in vivo antitumor efficacy of STA551, tumor-bearing mice were treated with Sta-MB and Ure-MB. Sta-MB has the same variable region as STA551 and has MB as the constant region, which is an engineered constant region of mouse IgG1 to increase binding activity to mouse Fc gamma receptor II (FcyRII). Ure-MB has urelumab-like Fab and MB as the constant region. Ure-MB was used as a conventional CD137 agonist antibody in contrast to Sta-MB, an anti-CD137 switch antibody ${ }^{6}$. To investigate anti-CD137 antibody binding in tissues, we administered Alexa Fluor 488-labeled Ure-MB and isotype control antibody (anti-KLH-MB) to tumor-bearing hCD137 KI mice. These antibodies were administered at the doses of $1 \mathrm{mg} / \mathrm{kg}$ twice. Sta-MB was not used because it might dissociate from the target during the process of ex vivo analysis. Ure-MB was detected not only in tumor but also in spleen (Fig. 3A-B). Ure-MB bound to $5-30 \%$ of $C D 45^{+}$cells in tumor and $10-25 \%$ of $C D 45^{+}$cells in spleen. In addition, Ure-MB bound to $C D 4^{+} T$ cells, $C D 8^{+} T$ cells and $C D 11 b^{+}$cells (Fig. $3 D$ ). NK cells in tumor were not detected in this experiment. In tumor, isotype control antibody bound to CD45+ cells, especially CD11b+ cells (Fig. 3B-C). CD137 expression was not evaluated because Ure-MB binds to the same site of CD137 as the detection antibody. Given that the Ure-MB binding population was consistent with the CD137 expressing population shown in Fig. 2B, Ure-MB would bind to CD137-expressing immune cells. Taken together, these results indicate that Ure-MB binds to targets in both tumor and normal tissues.

To verify the target binding of STA551 in vivo, we investigated the distribution of Sta-MB in tumor and spleen. Alexa Fluor 488-labeled Ure-MB, Sta-MB, and isotype control antibody were administered to tumorbearing hCD137 KI mice and detected by two-photon microscopy. In tumor, fluorescence from all antibodies were detected (Fig. 4A, C, Suppl Fig. 2). However, Sta-MB showed weaker fluorescence in spleen than Ure-MB (Fig. 4B, C), and the Sta-MB fluorescence was comparable to isotype control antibody. These data suggested that Sta-MB distributes and binds differently in tumor and spleen in vivo and shows little binding to CD137 in spleen. 
Table 1

Antibody concentration in plasma and tissues. Alexa Fluor 488-labeled isotype control antibody, Ure-MB or Sta-MB were administered twice at doses of $1 \mathrm{mg} / \mathrm{kg}$. Tissues were collected the day after the second administration. Antibody concentrations in plasma and tissue lysates were measured by an electrochemiluminescence (ECL) assay.

\begin{tabular}{|llllll|}
\hline antibody & \multicolumn{4}{l}{ concentration $(\mu \mathrm{g} / \mathrm{mL}$ plasma or $\mu \mathrm{g} / \mathrm{g}$ tissue $)$} & \multicolumn{1}{l|}{$\mathrm{T} / \mathrm{P}$} \\
\cline { 2 - 6 } & plasma & spleen & tumor & spleen & tumor \\
\hline Ure-MB & 4.7 & 1.2 & 1.1 & 0.26 & 0.25 \\
\hline Sta-MB & 5.5 & 0.5 & 0.6 & 0.09 & 0.11 \\
\hline isotype control & 8.0 & 1.5 & 5.0 & 0.19 & 0.62 \\
\hline
\end{tabular}

\section{Discussion}

In this study, we revealed that anti-CD137 switch antibody binds to target cells differently in tumor and spleen in vivo by using two-photon microscopy. We first examined the CD137 expression levels of tumor and spleen in tumor-bearing hCD137 KI mice. Conventional anti-CD137 antibody was then administered to tumor-bearing hCD137 KI mice to determine the distribution of the antibody to the tumor and spleen. Finally, two-photon microscopy was used to detect the distribution of anti-CD137 switch antibody to tumor and spleen in vivo.

To confirm the different binding ability of STA551 between tissues in vivo, we needed to detect the distribution of antibodies in a non-invasive way to minimize ATP-concentration changes in tissues. Therefore, we used two-photon microscopy to detect antibodies intravitally. In experiments using twophoton microscopy, it can be time-consuming to prepare animals, optimize imaging, and monitor animals. On the other hand, in ex vivo analysis using flow cytometry, several experimental conditions, such as dosing regimen, can be verified with better thruput. In addition, ex vivo studies can detect the expression of target molecules, the binding of antibodies, and identify the types of cells. Establishing optimal imaging conditions for intravital imaging by using ex vivo flow cytometric analysis would be useful in studying antibody distribution in vivo.

In this study, in vivo imaging revealed that Sta-MB distributed and bound to the cells in tumor but much less so in spleen. It has been suggested that the toxicity induced by anti-CD137 antibody is CD137 dependent, and that T-cell and macrophage infiltration and secreted cytokines by the cells are involved in the pathogenesis ${ }^{20-22}$. Ure-MB was highly distributed to not only tumor but also spleen, and it seemed to lead to systemic toxicity by inducing CD137 signaling to normal tissues. Our findings suggest that the tumor-selective target-binding ability of STA551 avoids the systemic reaction caused by conventional anti-CD137 antibodies.

Ure-MB, a conventional anti-CD137 antibody, bound to cells in tumor and spleen. Ure-MB mainly bound to T cells and NK cells, consistent with cell populations in which CD137 expression was observed Fig. 2B or 
previously reported ${ }^{23}$. Thus, this suggests that Ure-MB bound to the cells in a CD137 dependent manner. There were two possible reasons that the binding of Ure-MB was greater than the expression of CD137. The first reason is that the administration of antibody increased the expression of CD137 molecules. CD137 agonist signals are known to activate T cells and other immune cells, leading to increased expression of $C D 137^{24}$. Ure-MB administered to mice might bind to CD137, introduce CD137 agonist signals in tumor and spleen and induce increased expression of CD137 in tissues. The second reason is the Fc region-mediated binding of Ure-MB. Ure-MB, isotype control antibody and Sta-MB have engineered Fc regions that bind to murine FcyRs, particularly FcyRII ${ }^{6}$, and FcyRII is predominantly expressed in the myeloid lineage cells ${ }^{25}$. Isotype control antibody bound to $C D 45^{+}$cells, especially CD $11 \mathrm{~b}^{+}$cells, suggesting that the antibody bound to the cell via Fc region. However, the binding of Ure-MB to CD11 ${ }^{+}$ cells was comparable with that of isotype control antibody in either tumor or spleen, suggesting that Fabmediated binding is more prevalent. In addition, since the main Ure-MB binding cells were confirmed to be CD137 expressing cells, such as T cells and NK cells, binding of Ure-MB to cells was thought to be primarily Fab-mediated binding.

In the imaging experiment, fluorescence from all antibodies were detected in tumor. Table1 and suppl Fig. 1 show the concentration of Ure-MB, Sta-MB, and isotype control antibody in plasma, spleen, and tumor. The concentration of isotype control antibody in each tissue, especially in tumor, was higher than the other two antibodies. In image analysis, the difference could not be distinguished between the antibodies which were binding to target molecule and non-binding antibodies and/or bound nonspecifically in tissues. Isotype control antibody which was highly distributed to tumor might be detected as fluorescent signal in the imaging experiment.

STA551 is designed to bind to CD137 strongly in the presence of $100 \mu \mathrm{M}$ ATP but not in the absence of ATP $^{6}$. Murine ATP levels have been reported to be approximately $100 \mu \mathrm{M}$ of extracellular ATP in tumor and $10-100 \mathrm{nM}$ in normal tissues ${ }^{7,8,26}$. However, it is difficult to measure the exact ATP concentration in physiological conditions because ATP concentration changes depending on the sampling and measurement conditions due to degradation of ATP and release of intracellular ATP. This study revealed that Sta-MB showed binding in tumor and little binding to spleen. This data suggested that, in physiological conditions, tumor had ATP levels of $100 \mu \mathrm{M}$ or higher and normal tissue had lower ATP levels. The present imaging results may be useful for estimating ATP levels of tissues under physiological conditions. In addition, human ATP levels have been reported to be 10-100 nM in normal tissues ${ }^{26}$ and more than $10 \mu \mathrm{M}$ in tumor, and there is around 1,000-fold difference in ATP concentration between tumor and normal tissues, which is similar in mice ${ }^{27}$. Considering the similarity in ATP distributions in human and mouse tissues, it is anticipated that STA551 will also exhibit tumor-selective binding in humans. Thus, STA551 is expected to exert anti-tumor efficacy with tumor selective CD137 signals, while reducing systemic reaction, even in human patients.

In conclusion, we showed that STA551 distributes in tumor but little in spleen. Such STA551 distribution demonstrated more clearly the reason why STA551 work in tumor but not in normal tissues. Because of 
less distribution in normal tissues, STA551 could be a promising therapeutic antibody for patients with currently difficult-to-treat cancers.

\section{Methods \\ Cell line}

LLC1/OVA/hGPC3 cells were established by transfecting human GPC3 and chicken ovalbumin (OVA) expressing plasmids into LLC1, which was purchased from ATCC ${ }^{6,28}$.

\section{Animals}

The animal studies were carried out in compliance with the ARRIVE guidelines (https://arriveguidelines.org/). All animal studies were conducted in accordance with the animal treatment policy of the Institutional Animal Care and Use Committee (IACUC) at Chugai pharmaceutical Co., Ltd., and the Animal Experiments Committee of Osaka University. The animal experiments were performed in accordance with the Guidelines for the Care and Use of Laboratory Animals at Chugai Pharmaceutical Co., Ltd, which is accredited by the Association for Assessment and Accreditation of Laboratory Animal Care (AAALAC) International. All animal studies were approved by the Institutional Animal Care and Use Committee (IACUC) at Chugai pharmaceutical Co., Ltd., and the Animal Experiments Committee of Osaka University. To create a subcutaneous tumor model for the in vivo antibody distribution study, $1.0 \times 10^{6}$ LLC1/OVA/hGPC3 cells ${ }^{6}$ were subcutaneously inoculated into 8-week old hCD137 KI male mice ${ }^{6}$. hCD137 KI mice were generated by replacing mouse CD137 with human CD137, and did not have mouse CD137 gene and protein but human CD137 gene and protein ${ }^{6}$.

\section{Antibody labeling}

Alexa Fluor 488 Antibody Labeling Kit (Thermo Fisher Scientific) was used for labeling Ure-MB, Sta-MB, and isotype control antibody (anti-KLH-MB). The binding activity to human CD137 of labeled Ure-MB and Sta-MB in the presence and absence of ATP were determined by Biacore assay.

\section{in vivo study in hCD137 KI mice}

In vivo study was performed according to previously reported procedures ${ }^{29}$ with some modifications. About 3 weeks after LLC1/OVA/hGPC3 tumor inoculation, $1 \mathrm{mg} / \mathrm{kg}$ of Alexa Flour 488-labeled isotype control antibody or PBS were intravenously administered once to investigate CD137 expression. For antibody distribution study, $1 \mathrm{mg} / \mathrm{kg}$ of Alexa Flour 488-labeled antibodies were intravenously administered at day 0 and 3 . Mice were anesthetized with isoflurane at 24 hours after the administration to be observed by intravital two-photon microscopy or to have their plasma and tissues collected for single cell analysis and determining antibody concentrations in tissues. For determining concentrations in tissues, each tissue sample was homogenized with a TissueLyser II (Quiagen) in lysis buffer (Cell Signaling Technology \#9803) added with cOmplete ${ }^{\mathrm{TM}}$ Protease Inhibitor Cocktail (Roche \#04693116001). Homogenate was centrifuged at $14,000 \mathrm{rpm}$ for $15 \mathrm{~min}$ at $4^{\circ} \mathrm{C}$ and the supernatant was collected to be 
analyzed. Antibody concentrations in plasma and tissue lysate were measured by an electrochemiluminescence (ECL) assay using the MULTI-ARRAY Standard 384-well plates (Meso Scale Diagnostics) coated with recombinant human CD137 (Sino Biological) or Keyhole limpet hemocyanin (KLH) from Megathura crenulata (Sigma) and biotinylated anti-mouse IgG antibody (Southern Biotech). The ECL signals were detected by MESO SECTOR S 600 (Meso Scale Diagnostics) after adding the SULFO-TAG labeled Streptavidin (Meso Scale Diagnostics).

\section{Flow Cytometry}

For analyzing target expression and antibody distribution by flow cytometry, single cell suspensions of each excised organ were prepared according to the manufacture's protocol using the tumor dissociation kit (Miltenyi Biotec) or by dissociation using slide grass. After lysing the red blood cells by Pharm Lyse (BD Biosciences), cell suspensions were reconstituted by PBS containing $0.5 \%$ BSA and $2 \mathrm{mM}$ EDTA for staining cell surface and subcellular epitopes. Cell suspensions were stained with anti-CD 45 Per-CP-Cy5.5 (30-F11), anti-CD3e BUV496 (145-2C11), anti-CD4 BV786 (RM4-5), anti-CD8a PE-Cy7 (53-6.7), anti-CD19 APC (1D3), anti-CD11b APC-Cy7 (M1/70), anti-CD49b BV421 (DX5), anti-human CD137 PE (4B4-1) purchased from BD Biosciences, and zombie aqua (BioLegend). Following fixation, data were acquired on FACS LSRFortessa ${ }^{\mathrm{TM}} \mathrm{X}-20$ (BD Biosciences). Data analysis was performed using FlowJo software (version 10.2).

\section{Intravital two-photon microscopy imaging}

As pretreatment for intravital two-photon microscopy imaging, mice were shaved, and the hair was removed with depilatory cream to prevent it being mixed into the visual field. The spleen and tumor were then surgically exposed to be covered with the cover glass using n-butyl cyanoacrylate glue (3M Vetbond Tissue Adhesive, 3M). Qtracker 655 Vascular Labels (Thermo Fisher Scientific) were intravenously administered into mice just before imaging of spleen and tumor in order to visualize the blood vessels in these tissues. Inverted multiphoton microscope (A1R-MP, Nikon) equipped with multi-immersion objectives (20X, Plan Fluor, numerical aperture [NA], 0.75, Nikon) was used to observe tumor and spleen. The microscope was driven by a Chameleon Vision II Ti:Sapphire laser (Coherent) tuned to $930 \mathrm{~nm}$. The fluorescence was detected by an external non-descanned detector with four channels (Nikon) with three dichroic mirrors (495, 560, and $593 \mathrm{~nm}$ ) and four band-pass filters: $492 \mathrm{~nm}$ for the second harmonic generation (SHG) signal, $525 / 50 \mathrm{~nm}$ for Alexa Fluor $488,575 / 25 \mathrm{~nm}$ for tdTomato, and $629 / 56 \mathrm{~nm}$ for Qtracker 655. 4 to 11 images of around $300 \mu \mathrm{m} \times 300 \mu \mathrm{m}$ with a vertical step size of about $3 \mu \mathrm{m}$ to a depth of around $100 \mu \mathrm{m}$ were collected of each tissue and then analyzed by NIS-Elements integrated software (Nikon) to create the maximum intensity projection (MIP) images with median filters for noise reduction. The area of antibody-binding region in tumor and spleen was detected by ImageJ software. The antibody-binding region was extracted from MIP images. Area fraction was calculated by fluorescence area/total area in all images.

\section{Statistical Analysis}


Statistical analyses were performed with GraphPad Prism 7.0 (GraphPad Software). CD137 expression level and antibody distribution were compared by using Student t-test. Antibody fluorescence in the two groups were compared using Tukey's multiple comparisons test. Significant values were marked as: * $p<$ $0.05, * \star p<0.01, * \star * p<0.001$, and n.s.

\section{Declarations}

\section{Acknowledgements}

We thank our colleagues at Chugai Pharmaceutical Co., Ltd. and Chugai Research Institute for Medical Science Inc for their technical and scientific support. We also acknowledge Yuji Hori, Shinichirou Yamaki, Ayumi Maeno, Takuya Kato, Yasuhiro Oyama, Atsushi Ohba, Naoto Tanishiki, Akihisa Sakamoto, Masaaki Goto, Yohei Yamamoto, Akira Hayasaka, Shiho Suzuki, Meiri Kawazoe for creating and providing antibodies; Keiko Esaki, Meiri Kawazoe, Shoko Mochizuki for checking binding activity after fluorescent labeling; Koichi Jishage, Otoya Ueda, Naoko Wada for breeding hCD137 KI mice; Ayumi Maeda, Asuka Otsuka, Hiroki Fukui for maintaining LLC1/OVA/hGPC3 cell; Hiroaki Abe, Mayu Fujita for providing technical support for imaging experiments; Shigeto Seno for providing support for imaging analysis; and Jacob Davis for review of the manuscripts.

\section{Author contributions statement}

C.K. and H.T. performed experiments, analyzed the data, and wrote the manuscripts. K.O. and M.H. helped design the study, discussed the results and revised the manuscripts. K.H. provided support for imaging experiment and revised the manuscripts. J.K. and M.I. discussed the results and provided technical support or ideas. Y.N. helped design the study, provided support for in vivo experiments, discussed the results and revised the manuscripts. M.K.-S. helped design the study, discussed the results, revised the manuscripts and supervised the project. M.T. provided supervisory support. This work was partly supported by funding from Innovative Drug Discovery and Development Project (Japan Agency for Medical Research and Development [AMED] under Grant Number JP20am0401009 to M.I. and J.K.).

\section{Competing interests}

C.K., H.T., K.O., M.H., K.H., Y.N., M.K.-S., and M.T. are employees of Chugai Pharmaceutical Co., Ltd. Y.N. is an employee of Chugai Pharmabody Research Pte. Ltd. J.K. and M.I. receive research support from Chugai Pharmaceutical Co., Ltd. This study was funded by Chugai Pharmaceutical Co., Ltd.

\section{References}

1. DeBenedette, M. A. et al. Role of 4-1BB ligand in costimulation of T lymphocyte growth and its upregulation on M12 B lymphomas by cAMP. The Journal of experimental medicine 181, 985-992, doi:10.1084/jem.181.3.985 (1995). 
2. Vinay, D. S. \& Kwon, B. S. Immunotherapy of cancer with 4-1BB. Mol Cancer Ther 11, 1062-1070, doi:10.1158/1535-7163.MCT-11-0677 (2012).

3. Yonezawa, A., Dutt, S., Chester, C., Kim, J. \& Kohrt, H. E. Boosting Cancer Immunotherapy with AntiCD137 Antibody Therapy. Clinical cancer research: an official journal of the American Association for Cancer Research 21, 3113-3120, doi:10.1158/1078-0432.CCR-15-0263 (2015).

4. Segal, N. H. et al. Results from an Integrated Safety Analysis of Urelumab, an Agonist Anti-CD137 Monoclonal Antibody. Clinical cancer research: an official journal of the American Association for Cancer Research 23, 1929-1936, doi:10.1158/1078-0432.CCR-16-1272 (2017).

5. Segal, N. H. et al. Phase I Study of Single-Agent Utomilumab (PF-05082566), a 4-1BB/CD137 Agonist, in Patients with Advanced Cancer. Clinical cancer research: an official journal of the American Association for Cancer Research 24, 1816-1823, doi:10.1158/1078-0432.CCR-17-1922 (2018).

6. Kamata-Sakurai, M. et al. Antibody to CD137 Activated by Extracellular Adenosine Triphosphate Is Tumor Selective and Broadly Effective In Vivo without Systemic Immune Activation. Cancer discovery, doi:10.1158/2159-8290.CD-20-0328 (2020).

7. Di Virgilio, F. \& Adinolfi, E. Extracellular purines, purinergic receptors and tumor growth. Oncogene 36, 293-303, doi:10.1038/onc.2016.206 (2017).

8. Pellegatti, P. et al. Increased level of extracellular ATP at tumor sites: in vivo imaging with plasma membrane luciferase. PloS one 3, e2599, doi:10.1371/journal.pone.0002599 (2008).

9. de Andrade Mello, P., Coutinho-Silva, R. \& Savio, L. E. B. Multifaceted Effects of Extracellular Adenosine Triphosphate and Adenosine in the Tumor-Host Interaction and Therapeutic Perspectives. Frontiers in immunology 8, 1526, doi:10.3389/fimmu.2017.01526 (2017).

10. Levine, A. \& Markowitz, O. Introduction to reflectance confocal microscopy and its use in clinical practice. JAAD Case Rep 4, 1014-1023, doi:10.1016/j.jdcr.2018.09.019 (2018).

11. Helmchen, F. \& Denk, W. Deep tissue two-photon microscopy. Nature methods 2, 932-940, doi:10.1038/nmeth818 (2005).

12. Helmchen, F. \& Denk, W. New developments in multiphoton microscopy. Curr Opin Neurobio/ 12, 593601, doi:10.1016/s0959-4388(02)00362-8 (2002).

13. Zinselmeyer, B. H. et al. Chapter 16. Two-photon microscopy and multidimensional analysis of cell dynamics. Methods Enzymol 461, 349-378, doi:10.1016/S0076-6879(09)05416-0 (2009).

14. Borjini, N., Paouri, E., Tognatta, R., Akassoglou, K. \& Davalos, D. Imaging the dynamic interactions between immune cells and the neurovascular interface in the spinal cord. Exp Neurol 322, 113046, doi:10.1016/j.expneurol.2019.113046 (2019).

15. Hasegawa, T., Kikuta, J. \& Ishii, M. Imaging the Bone-Immune Cell Interaction in Bone Destruction. Frontiers in immunology 10, 596, doi:10.3389/fimmu.2019.00596 (2019).

16. Svoboda, K. \& Yasuda, R. Principles of two-photon excitation microscopy and its applications to neuroscience. Neuron 50, 823-839, doi:10.1016/j.neuron.2006.05.019 (2006). 
17. Hasegawa, T. \& Ishii, M. Visualizing bone tissue in homeostatic and pathological conditions. Proc Jpn Acad Ser B Phys Biol Sci 96, 43-49, doi:10.2183/pjab.96.004 (2020).

18. Sahu, P. \& Mazumder, N. Advances in adaptive optics-based two-photon fluorescence microscopy for brain imaging. Lasers Med Sci 35, 317-328, doi:10.1007/s10103-019-02908-z (2020).

19. Ng, L. G., Mrass, P., Kinjyo, I., Reiner, S. L. \& Weninger, W. Two-photon imaging of effector T-cell behavior: lessons from a tumor model. Immunol Rev 221, 147-162, doi:10.1111/j.1600065X.2008.00596.x (2008).

20. Dubrot, J. et al. Treatment with anti-CD137 mAbs causes intense accumulations of liver T cells without selective antitumor immunotherapeutic effects in this organ. Cancer immunology, immunotherapy: C/I 59, 1223-1233, doi:10.1007/s00262-010-0846-9 (2010).

21. Lin, G. H., Snell, L. M., Wortzman, M. E., Clouthier, D. L. \& Watts, T. H. GITR-dependent regulation of 41BB expression: implications for T cell memory and anti-4-1BB-induced pathology. Journal of immunology 190, 4627-4639, doi:10.4049/jimmunol.1201854 (2013).

22. Niu, L. et al. Cytokine-mediated disruption of lymphocyte trafficking, hemopoiesis, and induction of lymphopenia, anemia, and thrombocytopenia in anti-CD137-treated mice. Journal of immunology 178, 4194-4213, doi:10.4049/jimmunol.178.7.4194 (2007).

23. Palazon, A. et al. The HIF-1 alpha hypoxia response in tumor-infiltrating $T$ lymphocytes induces functional CD137 (4-1BB) for immunotherapy. Cancer discovery 2, 608-623, doi:10.1158/21598290.CD-11-0314 (2012).

24. Makkouk, A., Chester, C. \& Kohrt, H. E. Rationale for anti-CD137 cancer immunotherapy. Eur J Cancer 54, 112-119, doi:10.1016/j.ejca.2015.09.026 (2016).

25. Bruhns, P. \& Jonsson, F. Mouse and human FcR effector functions. Immunol Rev 268, 25-51, doi:10.1111/imr.12350 (2015).

26. Gorman, M. W., Feigl, E. O. \& Buffington, C. W. Human plasma ATP concentration. Clin Chem 53, 318325, doi:10.1373/clinchem.2006.076364 (2007).

27. Hu, L. P. et al. Targeting Purinergic Receptor P2Y2 Prevents the Growth of Pancreatic Ductal Adenocarcinoma by Inhibiting Cancer Cell Glycolysis. Clinical cancer research: an official journal of the American Association for Cancer Research 25, 1318-1330, doi:10.1158/1078-0432.CCR-18-2297 (2019).

28. Ishiguro, T. et al. An anti-glypican 3/CD3 bispecific T cell-redirecting antibody for treatment of solid tumors. Science translational medicine 9, doi:10.1126/scitranslmed.aal4291 (2017).

29. Mimoto, F. et al. Exploitation of Elevated Extracellular ATP to Specifically Direct Antibody to Tumor Microenvironment. Cell reports 33, 108542, doi:10.1016/j.celrep.2020.108542 (2020).

\section{Figures}




\section{STEP1}

\section{Analysis of human CD137 knock-in mouse}

human CD137 expression in tumor and normal tissues

CD137

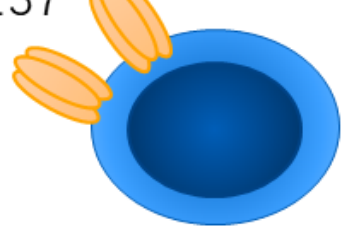

STEP2

anti-CD137 antibody

Anti-CD137 antibody distribution

Antibody binding in tumor and normal tissues

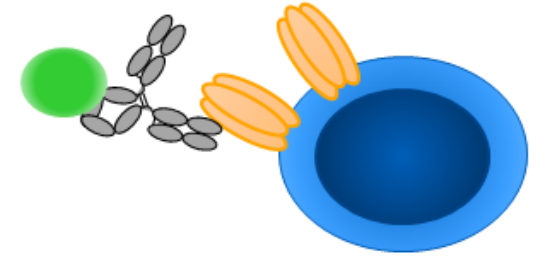

\section{STEP3}

Intravital imaging of antibody

Antibody detection by two-photon microscopy

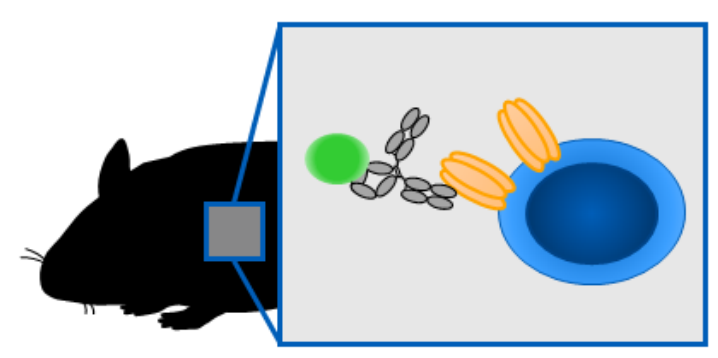

Figure 1

Research strategy for detection of antibody binding in tissues by two-photon microscopy. In step1, human CD137 expression was examined in tumor-bearing hCD137 KI mice. In step2, fluorescent labeled anti-CD137 antibody was administered to hCD137 KI mice. Antibody binding cells in tumor and spleen were detected. Finally, antibody binding cells in tumor and spleen were detected by two-photon microscopy in step3. 

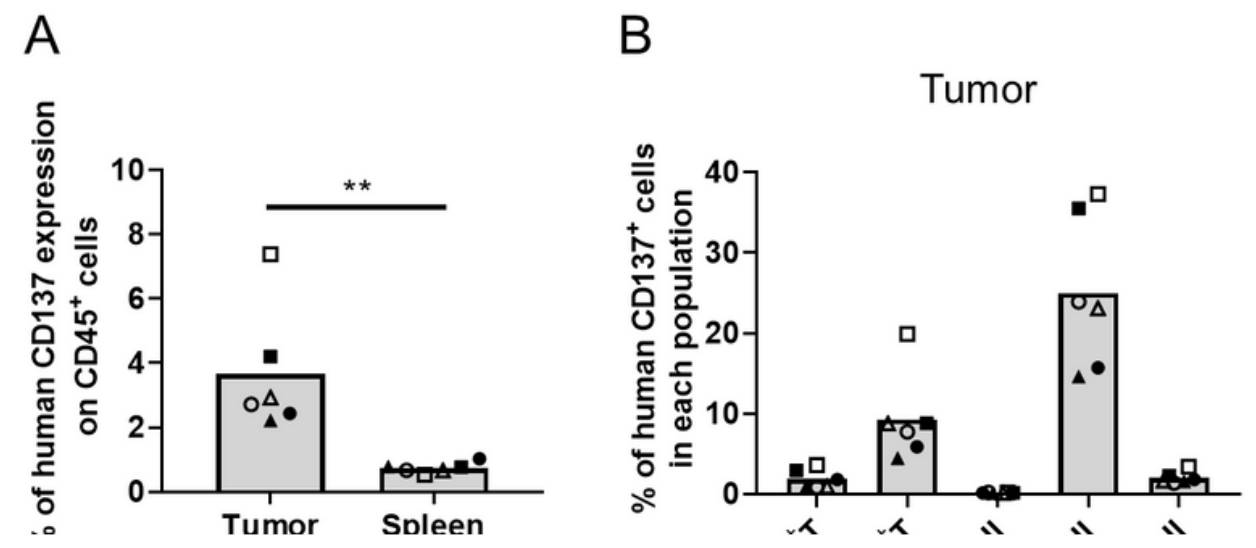

Spleen

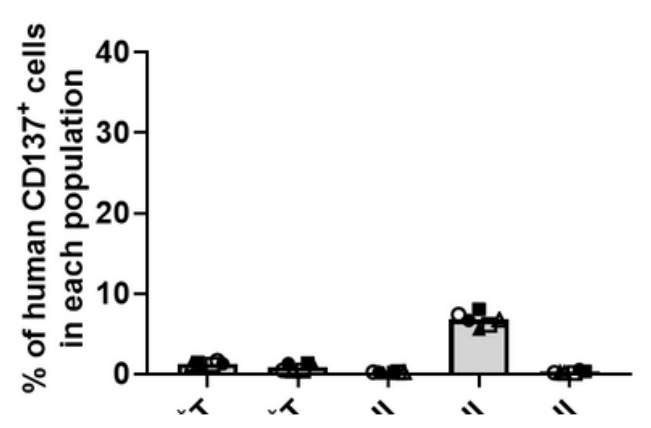

\section{Figure 2}

Human CD137 expression in LLC1/OVA/hGPC3-bearing hCD137 KI mice. Tumor and spleen were sampled at 24 hours after administration of PBS (closed symbols) or isotype control antibody (open symbols). (A) human CD137 expression on CD45 positive cells in tumor and spleen. Individual values and mean in each tissue are shown. ${ }^{*} \mathrm{P}<0.01$ by $t$ test. (B) human CD137 expression on each type of cell. The percentage of human CD137 positive cells for each cell type was calculated by flow cytometry. Individual values and mean in each cell type are shown.

\section{Figure 3}

Anti-human CD137 antibody binding cells in LLC1/OVA/hGPC3-bearing hCD137 KI mice. Alexa Fluor 488labeled isotype control antibody or Ure-MB were administered twice at doses of $1 \mathrm{mg} / \mathrm{kg}$. The tissues were sampled the day after the second administration. (A) Representative plotting data of Alexa Fluor 488-labeled antibody binding CD45 positive cells in tissues. The population in the square represents Alexa Fluor 488 positive cells. (B) The percentage of Alexa Fluor 488-labeled antibody binding cells in CD45 positive cells. Alexa Fluor 488 positive cells were detected in each tissue. The data was collected from three mice in each group. ${ }^{\star} \mathrm{P}<0.05$ by $\mathrm{t}$ test. (C, D) (C)Alexa Fluor 488-labeled isotype control antibody binding and (D) Alexa Fluor 488-labeled Ure-MB binding on each type of cells in tumor and spleen. The percentage of Alexa Fluor 488 positive cells for each cell type was calculated by flow cytometry. \#NK cells in tumor were not detected in this experiment.

\section{Figure 4}


Detection of switch and non-switch antibody binding cells by two-photon microscopy. Alexa Fluor 488labeled isotype control antibody, Ure-MB or Sta-MB were administered twice at doses of $1 \mathrm{mg} / \mathrm{kg}$.

Intravital imaging by two-photon microscopy was conducted the day after second administration. (A-B) Representative images of Alexa Fluor 488-labeled antibody distribution in (A) tumor and (B) spleen. To visualize blood vessels, Qtracker 655 vascular labels were intravenously administered just before observation of two-photon microscopy. Green, Alexa Fluor 488-labeled antibody; magenta, blood vessels; blue, collagen fiber. (C) Quantitative analysis of Alexa Fluor 488-labeled antibody fluorescence. Antibodybinding region in tumor and spleen was detected from images of three or four mice. ${ }^{* \star} P<0.01,{ }^{* \star *} P<0.001$ by Tukey's multiple comparisons test.

\section{Supplementary Files}

This is a list of supplementary files associated with this preprint. Click to download.

- SupplementaryfigurelnvivoimagingofantiCD137switchantibody.pdf 